\title{
TRATAMIENTO DE REABSORCIÓN RADICULAR INTERNA PERFORANTE MEDIANTE USO DE SUSTITUTO DENTINARIO BIOCERÁMICO Y RECONSTRUCCIÓN CORONARIA CONRESINAS BULK FILL: REPORTE DE CASO

\author{
TREATMENT OF INTERNAL PERFORATING ROOT RESORPTION USING \\ BIOCERAMIC DENTIN SUBSTITUTE AND CORONARY RECONSTRUCTION WITH \\ BULK FILL RESINS: CASE REPORT
}

Nerea Yaringaño-Medina1,d, Jorge Alamo-Palomino1,a,c, Hugo García-Rivera1,b,c

\section{RESUMEN}

Se relata el tratamiento de conductos no convencional en la pieza 4.6 que presenta reabsorción radicular interna perforante, la cual compromete la raíz distal, el uso de un cemento sustituto dentinario biocerámico llamado Biodentine como relleno de dicho defecto y su posterior rehabilitación oral con resina condensable Bulkfill. Paciente se encuentra con pieza dental en función y asintomática.

Palabras clave: Reabsorción radicular, Tomografía computarizada. (Fuente: DeCS BIREME).

\begin{abstract}
Nonconventional duct treatment is reported in item 4.6 which shows internal perforating root resorption, which compromises the distal root, the use of a bioceramic dentin substitute cement called Biodentine as filling of said defect and its subsequent oral rehabilitation with Bulkfill condensable resin, patient is found with dental piece in function and asymptomatic.
\end{abstract}

Key words: Root resorption, tomography. (Source: MeSH NLM)

${ }^{1}$ Facultad de Odontología. Universidad de San Martín de Porres. Lima, Perú. aDoctor en Odontología

${ }^{b}$ Maestro en docencia universitaria

'Especialista en Carielogía y Endodoncia

${ }^{\mathrm{d}}$ Residente de la especialidad en Endodoncia

Correspondencia:

Nerea Yaringaño Medina

Dirección: Jr.Federico Villarreal 130 dpto 102 Breña-Lima

Correo electrónico: nerea-ym@hotmail.com
Este es un artículo Open Access distribuido bajo la licencia Creative Commons Atribución-NoComercialCompartirlgual 4.0

\section{(ㄷ)(이()}

\footnotetext{
Citar como: Yaringaño-Medina, Nerea, Alamo-Palomino Jorge , García-Rivera Hugo. Tratamiento de reabsorción radicular interna perforante mediante uso de sustituto dentinario biocerámico y reconstrucción coronaria con resinas Bulk Fill: Reporte de caso. KIRU. 2017;14(2):172-177. https://doi.org/10.24265/kiru.2017.v14n2.10
} 


\section{INTRODUCCIÓN}

Las reabsorciones radiculares internas son estadios patológicos multifactoriales que provocan la aparición de células clásticas multinucleadas en el tejido pulpar capaces de destruir las paredes de dentina. Se presenta en ocasiones como un problema idiopático ${ }^{(1-}$ 3). Son lesiones asintomáticas, respondiendo positivo a los test de sensibilidad pulpar. Clínicamente muestran la presencia de una mancha rosa, por presencia de tejido de granulación interno. En la radiografía hay presencia de agrandamiento del espacio del conducto radicular ${ }^{(1)}$. Son lesiones halladas en radiografías de manera casual(4-6). La presencia de dicha lesión es mejorada en la tomografía Cone beam que presenta muchas ventajas en comparación con la convencional película radiográfica, mostrando ubicación y extensión de la lesión ${ }^{(4,7-8)}$. Algunos pacientes desarrollan una tendencia hacia la reabsorción radicular. Como entidad representa uno de los más serios, irreversibles e irreparables riesgos del tratamiento dental(3). Existen materiales como el MTA, el cual favorece la reparación de dichas lesiones debido a sus propiedades de biocompatibilidad, buen sellado, bactericida, radiopacidad, osteogénico, cementogénico y regenerador de la nueva inserción del ligamento periodontal ${ }^{(4,9-12)}$. Actualmente nuevas fórmulas de silicato de calcio han sido introducidas como sustituyente dentinario, como es el caso de Biodentine (Septodont-Francia), el cual está formado por silicato tricálcico, carbonato cálcico y óxido de zirconio $^{(13)}$. Su biocompatibilidad e interesante respuesta biológica reparadora a nivel de la pulpa y tejidos periapicales hacen de este material una alternativa apropiada para recubrimientos pulpares, pulpotomías, reparador de perforaciones, en apexificaciones y relleno en el conducto radicular, favoreciendo un medio propicio para la reparación ${ }^{(14-}$

Dado que las piezas dentales con reabsorción radicular interna presentan un pronóstico reservado, se pueden considerar alternativas de tratamiento restaurador no convencional, como es el caso de las resinas Bulk Fill. Estas son resinas fotoactivadas de baja contracción, diseñadas para su colocación directa en la preparación cavitaria bajo la técnica monoincremental, sobre todo en piezas posteriores ahorrando tiempo en su aplicación (18-20).

\section{CASO CLÍNICO}

Paciente de sexo femenino, de 37 años, que llega a la Clínica Especializada en Odontología de la Universidad de San Martin de Porres, la cual se encuentra aparentemente en buen estado de salud. general (ABEG, ABEN, ABEH) y LOTEP. No refiere antecedente patológico de consideración y alergias. (Figura 1)
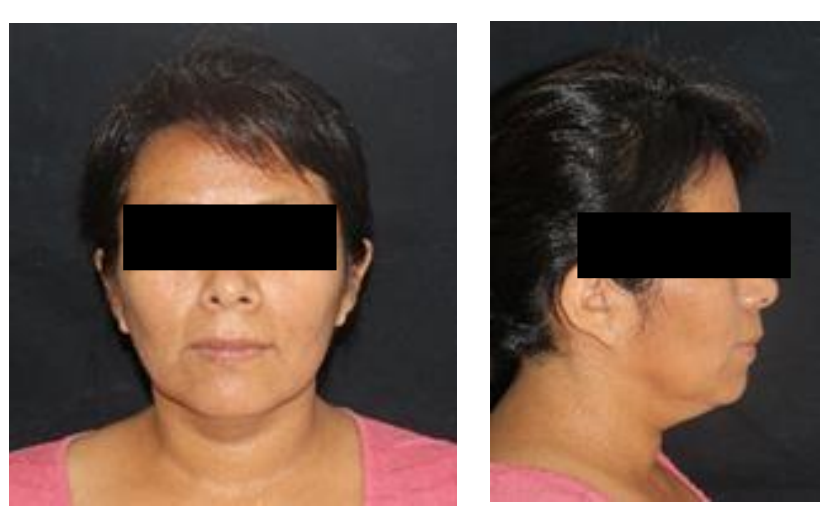

\section{Fiqura 1. Secuencia fotográfica extraoral.}

Al examen clínico intraoral presenta mucosa y encías conservadas y amplia lesión con aparente compromiso pulpar a nivel de la pieza 4.6, manifestando respuesta positiva a la prueba de sensibilidad al frío y calor.

Diagnóstico presuntivo: Pulpitis irreversible asintomática (Figura 2).
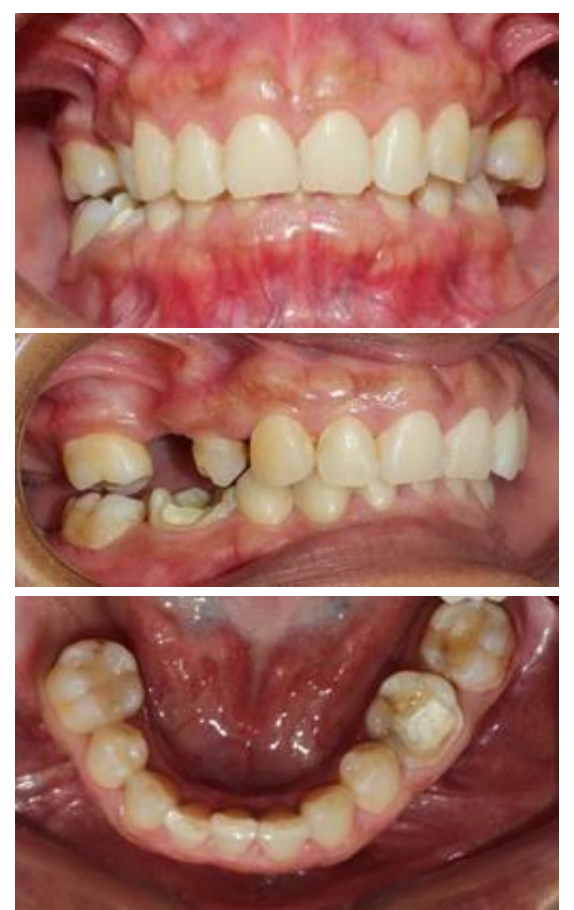

Figura 2. Secuencia fotográfica intraoral pieza № 4.6 .

Se decide tomar radiografía periapical para evaluar condición pulpar y periapical, decidiéndose realizar el tratamiento de conductos correspondiente (Figura 3). 


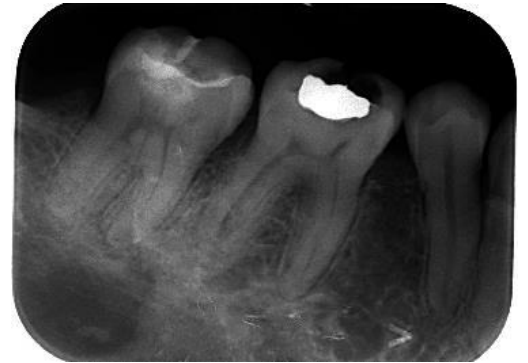

Figura 3. Radiografía periapical de diagnóstico.

Se realizó la apertura cameral y excéresis pulpar correspondiente e irrigación con hipoclorito de sodio al 2,5\%. La conductometría fue hecha con limas $\mathrm{K}$ $\mathrm{N}^{\circ} 25$ en los conductos mesiales y distal con lima $\mathrm{K}$ N³5 (Figura 4), decidiéndose colocar hidróxido de calcio durante dos semanas por sangrado abundante del conducto distal.

En la segunda cita, en la conometria se observó dificultad para la adaptación del cono maestro en

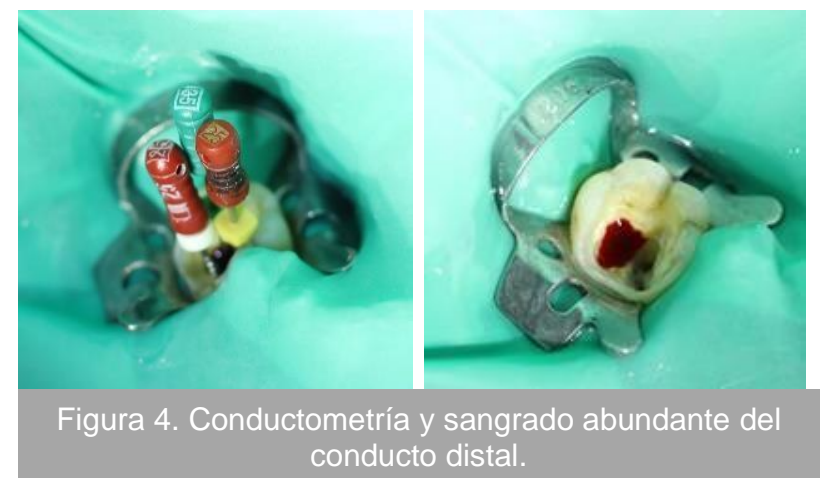

el conducto distal, notándose pérdida en la continuidad del conducto distal, sospechándose reabsorción radicular interna, por lo que se le sugirió la tomografía Cone beam a campo reducido confirmándose dicha presunción (Figura 5).

Diagnóstico definitivo: Pulpitis irreversible asintomática con reabsorción radicular interna.

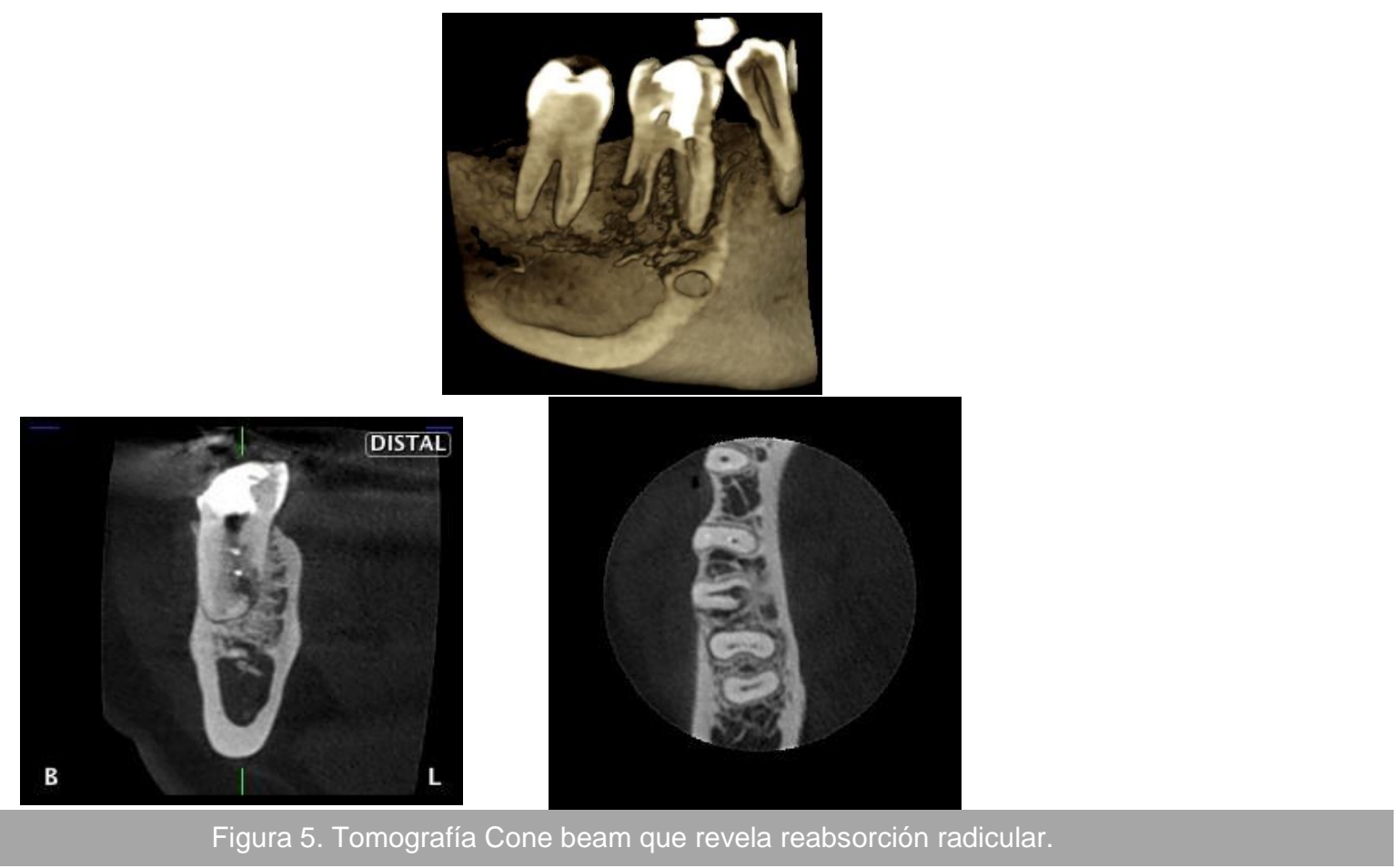

Se plantearon diferentes tratamientos en la evaluación por las áreas de rehabilitación y periodoncia, los cuales no eran favorables. Previo conocimiento y firma del consentimiento informado, se le planteó al paciente hacer un relleno del conducto distal con cemento sustituto dentinario biocerámico "Biodentine" (Francia-Septodont). El paciente quedó con pasta 3mix-MP como medicación intracanal durante 2 semanas al evidenciar en la tomografía lesión perirradicular a nivel de los conductos mesiales (Figura 6). 


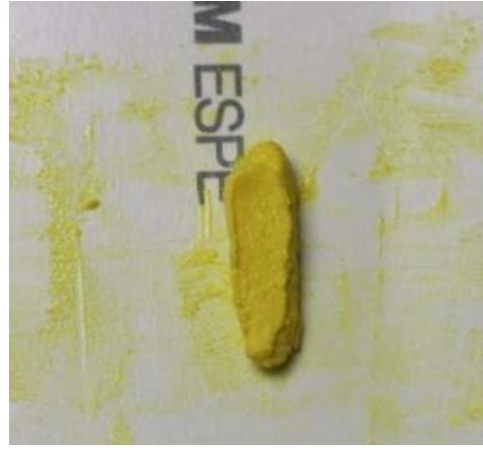

Figura 6. Pasta 3Mix-MP

Luego de dos semanas se realizó el abordaje clínico en 2 momentos: el primero fue la obturación de los conductos mesiales con técnica de termocompactación con uso de gutacondensor N55 (Dentsply Maillefer-Suiza) (Figura 7).
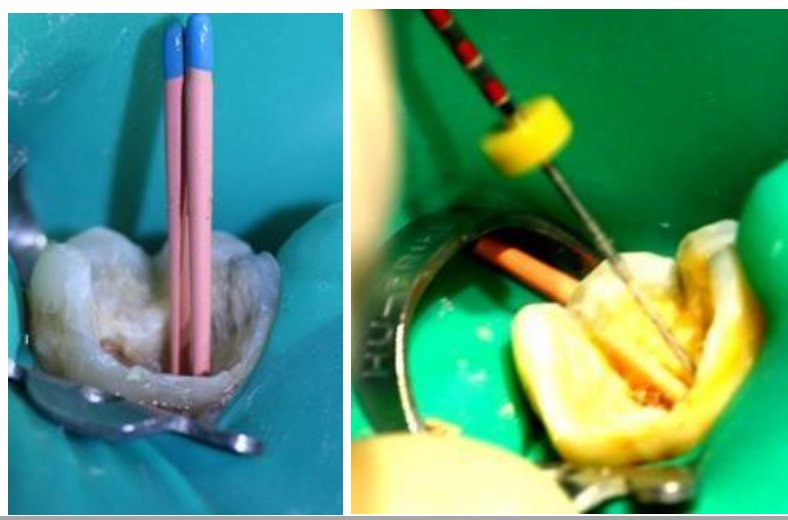

Figura 7. Termocompactación en conductos mesiales.

En el segundo momento se procedió a tratar el conducto distal, provocándose el sangrado para eliminar tejido de granulación con el uso de una punta de ultrasonido K30 (Satelec-Francia).

Se procedió a lavar el conducto con hipoclorito de sodio al $0,5 \%$ con 3 jeringas de $20 \mathrm{ml}$ cada una logrando parar el sangrado. Luego se secó el canal y se realizó la obturación con cemento biocerámico Biodentine (Septodont-Francia), el cual fue combinado durante 30 segundos a 3000rpm en un amalgamador calibrado por la casa Septodont. El material fue llevado al conducto con un porta amalgama y la compactación fue hecha con compactadores palmares de Machtou $N^{\circ} 3-4: 0.8$ $1.0 \mathrm{~mm}$ (VDW-Alemania) (Figura 8).

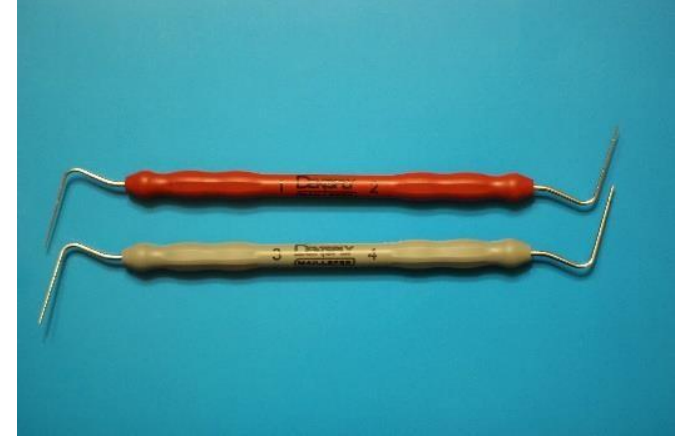

Figura 8. Compactadores palmares de Machtou

Luego fue vibrado dicho relleno en el conducto distal con punta ultrasónica inserto en el p5 Newtron XS (Satelec-Francia) para homogenizar el relleno (Figura 9).

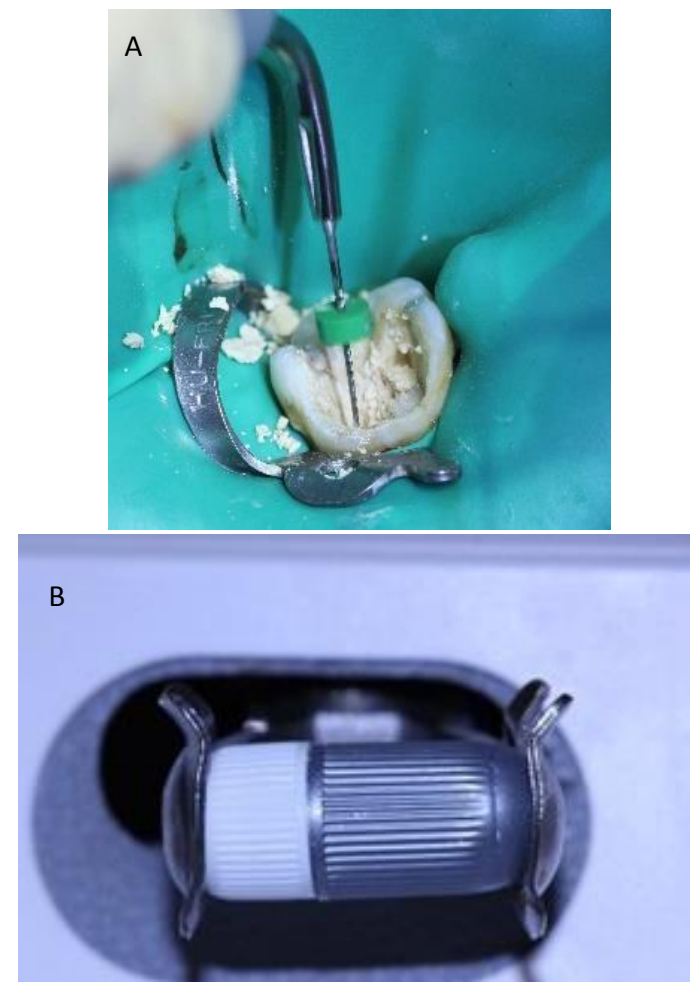

Figura 9. A y B. Vibración del biocerámico con ayuda del ultrasonido

Luego de 5 minutos, se observó el fraguado del material biocerámico. La rehabilitación de la pieza fue realizada con trozos de poste de fibra de vidrio Reforpost $\quad N^{\circ} 1$ (Angelus-Brasil), colocados horizontalmente en una base de resina Bulk fill flow 3M ESPE (Figura 10) y la reconstrucción coronaria se realizó con resina restauradora Bulk fill filtek (3M ESPE). Se realizó tomografía de control.

Paciente se mantiene asintomático hasta la fecha: 4 meses (Figura 11). 

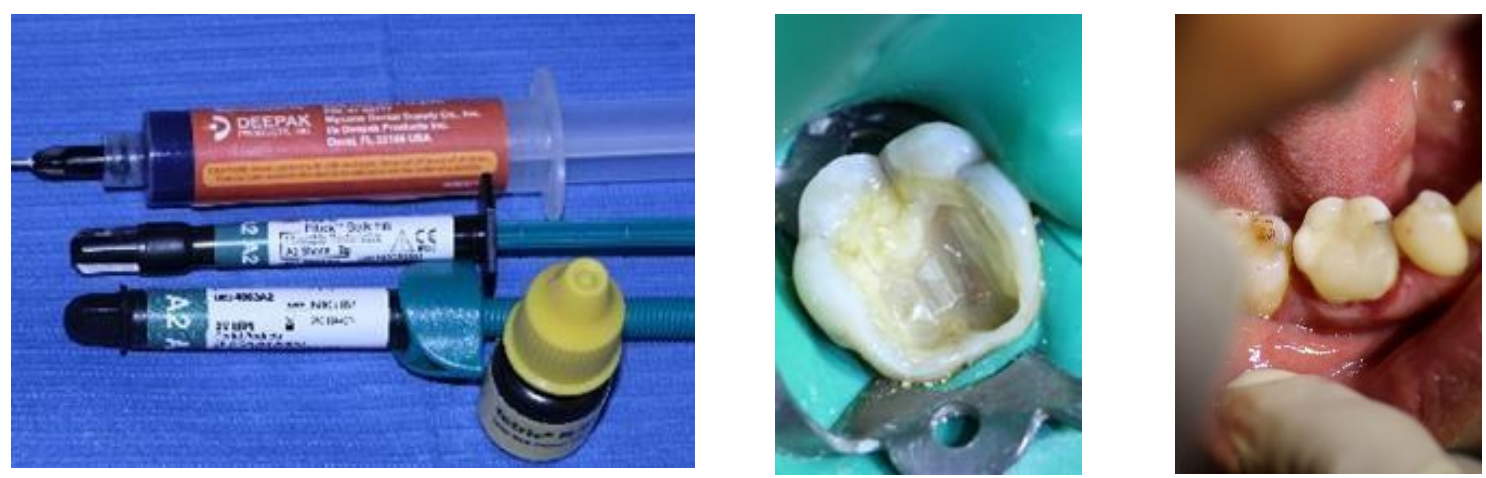

Figura 10. Reconstrucción de la pieza 4.6 con resina Bulk fill flow y condensable.

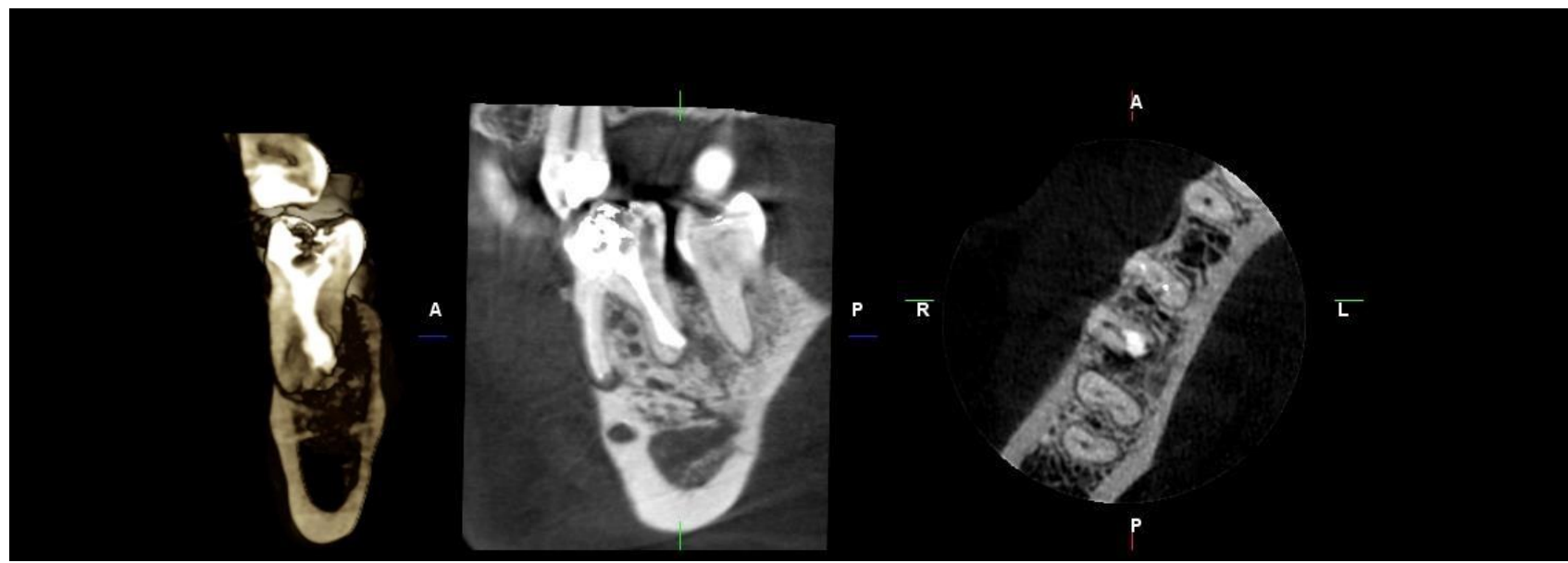

Figura 11. Control a 4 meses se mantiene asintomático y en función.

\section{DISCUSIÓN}

La reabsorción radicular interna es una patología pulpar crónica silenciosa que genera el debilitamiento radicular interno, el cual provoca que la pieza dental presente un pronóstico reservado ${ }^{(1-5,12)}$. Actualmente existen materiales que rellenan y refuerzan dichos defectos, como es el caso del MTA ${ }^{(6,9,10,11,15-17)}$ y cementos biocerámicos como Biodentine. Este último al tener propiedades osteogénicas y cementogénicas promueve la reparación de lesiones tales como perforaciones, cierres apicales a través de barreras y es sustituto de dentina en casos de reabsorciones radiculares internas y externas ${ }^{(13,14)}$. El presente caso muestra el uso del cemento biocerámico Biodentine como relleno de una reabsorción radicular interna, la cual fue evidenciada en toda su magnitud durante la tomografía computarizada Cone Beam ${ }^{(7-8)}$, ya que al ser un método moderno se pueden obtener imágenes tridimensionales, evidenciándose las lesiones en toda su magnitud y extensión, así como las lesiones osteolíticas periapicales. El uso final de resinas monoincrementales favorece la rehabilitación como fue descrito en el presente caso clínico, demostrando que es una excelente opción a las resinas core o núcleo y restauraciones indirectas ${ }^{(18-20)}$. Finalmente, el paciente se mantiene en observación y asintomático.

\section{CONCLUSIONES}

El uso del material sustituto dentinario biocerámico Biodentine favorece el tratamiento de reabsorciones radiculares internas junto con la rehabilitación coronaria oportuna. 
Fuentes de Financiamiento:

Autofinanciado

\section{Conflictos de Interés:}

Los autores declaran no tener conflicto de interés en la publicación de este reporte de caso.

\section{REFERENCIAS BIBLIOGRÁFICAS}

1. Levin L, Alan S. Law A, Holland G, Abbott P, Roda R. Identify and Define All Diagnostic Terms for Pulpal Health and Disease States. J Endod. 2009; 35: 16451657.

2. Levin L, Trope M. Root resorption. En: Hargreaves KM, Goodis HE, eds. Seltzer and Bender's dental pulp. Chicago: Quintessence; 2002: 425-47.

3. Presilla $\mathrm{M}$, Long $\mathrm{M}$, Betancourt I, Andreull B. Reabsorción radicular externa: presentación de un caso. Rev. Arch Med Camagüey. 2015;19(4): 383-9.

4. Bendyk-Szeffer M, Lagocka R, Trusewicz M, Lipski M, Buczkowska-Radlinska J. Perforating Internal Root Resorption Repaired with Mineral Trioxide Aggregate Caused Complete Resolution of Odontogenic Sinus Mucositis: A Case Report. J Endod. 2015; 41: 274278.

5. Patel S, Ricucci D, Durak C, Tay F. Internal root resorption: a review. J Endod. 2010; 36: 1107-21.

6. Altundasar E, Demir B. Management of a perforating internal resorptive defect with mineral trioxide aggregate: a case report. J Endod. 2009; 35(10): 1441-4.

7. Ball RL, Barbizam JV, Cohenca N. Intraoperative endodontic applications of conebeam computed tomography. J Endod. 2013; 39(4): 548-57.

8. Shemesh H, Cristescu RC, Wesselink PR, et al. The use of cone-beam computed tomography and digital periapical radiographs to diagnose root perforations. J Endod 2011; 37(4): 513-6.

9. Bogen $G$, Kuttler S. Mineral trioxide aggregate obturation: a review and case series. J Endod. 2009; 35(6): 777-90.

10. Torabinejad M, Parirokh M. Mineral trioxide aggregate: a comprehensive literature review - Part II: leakage and biocompatibility investigations. J Endod. 2010; 36(2): 190-202.

11. Parirokh $\mathrm{M}$, Torabinejad $\mathrm{M}$. Mineral trioxide aggregate: a comprehensive literature review-part III: clinical applications, drawbacks, and mechanism of action.J Endod. 2010; 36(3): 400-13.

12. Borkar S, Noronha de Ataide I. Management of a Massive Resorptive Lesion with Multiple Perforations in a Molar: Case Report. J Endod. 2015; 41(5): 753758.

13. Tziafa C, Koliniotou-Koumpia E, Papadimitriou S, Tziafas D. Dentinogenic Activity of Biodentine in Deep Cavities of Miniature Swine Teeth. J Endod. 2015; 41: 1161-1166.

14. Chen I, Salhab I, Seltzer F, Kim S, Duck Nah H. A New Calcium Silicate based Bioceramic Material Promotes Human Osteo and Odontogenic Stem Cell Proliferation and Survival via the Extracellular Signal-regulated
Kinase Signaling Pathway. J Endod. 2016; 42: 480486.

15. Ford TR, Torabinejad M, Abedi $H$. Using mineral trioxide aggegate as pulp capping material. J Am Dent Assoc. 1996; 127(10):1491-4.

16. Yildirim T, Gencoglu N, Firat I. Histologic study of furcation perforations treated with MTA or Super EBA in dogs's teeth. Oral Surg Oral Med Oral Pathol Oral Radiol Endod. 2005; 100: 120-4.

17. Baek SH, Plenk H, Kim S. Periapical tissue responses and cementum regeneration with amalgam, SuperEBA, and MTA as root-end filling materials. J Endod. 2005; 31(6): 444-9.

18. Domínguez R, Corral D, Bader M. Análisis comparativo in vitro del grado de sellado marginal de restauraciones de resina compuesta realizadas con un material monoincremental (Tetric n-ceram bulk fill), y uno convencional (Tetric n-ceram). Revista Dental de Chile. 2015; 106(1): 15-9.

19. Benetti AR, Havndrup-Pedersen C, Honoré D, Pedersen MK, Pallesen U. Bulk-fill resin composites: polymerization contraction, depth of cure, and gap formation. Oper Dent. 2015 Mar-Apr;40(2):190-200. doi: 10.2341/13-324-L. Epub 2014 Sep 11.

20. Saravia M. Resinas Bulk fill. ¿Cuándo, cómo y por qué? Lima; Glaxo Smith Kline Perú S.A; 2017. 